\title{
LO Phonon Renormalization in Optically Excited CuCl Nanocrystals
}

\author{
Lev Zimin, ${ }^{1}{ }^{*}$ Selvakumar V. Nair, ${ }^{1}$ and Yasuaki Masumoto ${ }^{1,2}$ \\ ${ }^{1}$ ERATO Single Quantum Dot Project, Japan Science and Technology Corporation, \\ 5-9-9 Tohkohdai, Tsukuba 300-2635, Japan \\ ${ }^{2}$ Institute of Physics, University of Tsukuba, Tsukuba 305-8571, Japan
}

(Received 15 December 1997)

\begin{abstract}
We have revealed sharp structures in the absorption spectrum of $\mathrm{CuCl}$ nanocrystals using persistent hole-burning spectroscopy and observed sidebands due to phonon-assisted absorption. The LO phonon frequency in the excited state of the nanocrystal is reduced by about $10 \%$ from the free value due to mixing with the exciton. When the mixing becomes resonant, the phonon sideband anticrosses with the excited exciton state. We present a theory of the phonon renormalization in nanocrystals and obtain results in excellent agreement with the experiment. [S0031-9007(98)05718-4]

PACS numbers: 63.22. $+\mathrm{m}, 61.46 .+\mathrm{w}, 71.24 .+\mathrm{q}, 71.35 .-\mathrm{y}$
\end{abstract}

Semiconductor nanocrystals being small structures containing only a few thousands of atoms, the absorption of a single photon would be expected to cause a measurable change in the elementary excitations of the nanocrystal. This exciting possibility is technologically attractive, fundamentally interesting, and poses new challenges to experimental studies. The size-selective studies of the excited state properties of the single nanoparticle are still at an early stage and most experimental efforts have been focused on the excited state absorption using pump-probe techniques $[1,2]$. Studies of lattice vibrations of nanocrystals have so far mostly focused on the ground state vibrational modes. Thus, LO phonons $[3,4]$ and confined acoustic phonons [5] have been observed in Raman scattering experiments, while exciton-phonon complexes have been observed in luminescence experiments [6]. However, an investigation of the exciton-phonon coupling and of the changes in the vibrational spectrum on excitation, a known feature of molecular systems and localized states in solids [7], is still lacking in nanocrystals. In this paper, we report the first observation of the excited state phonons in $\mathrm{CuCl}$ nanocrystals in phonon-assisted absorption, and show that the exciton-phonon coupling causes a strong renormalization of the LO phonons leading to a softening of the phonon frequency. The experimental results are obtained by means of persistent spectral hole burning and luminescence spectroscopies and are analyzed theoretically by a nonperturbative calculation of the phonon self-energy.

The experimental measurements were done on $\mathrm{CuCl}$ nanocrystals embedded in glass and crystalline $\mathrm{NaCl}$ matrices. The average radii, $R$, of the nanocrystals were controlled by heat treatment and were determined by small-angle x-ray scattering. A narrow-band dye laser (Lambda Physik; Scanmate 2EX) pumped by a $\mathrm{XeCl}$ excimer laser (Lambda Physik; Compex 110) was used as the pump source. The spectral line width of the dye laser was $0.15 \mathrm{~cm}^{-1}(0.019 \mathrm{meV})$ and the pulse duration was approximately $5 \mathrm{~ns}$. A halogen lamp was chosen as the probe source. As the decay time of the burned hole was of the order of a few hours [8], the persistent spectral hole burning (PSHB) experiment was done as follows. First, the absorption spectrum was obtained and then the sample was exposed to dye laser pulses to burn a persistent spectral hole. Then the laser exposure was stopped and the absorption spectrum was measured again. The subsequent measurements were done after erasing the burned spectral holes by exposing the sample to the ultraviolet light from an excimer laser or a deuterium lamp [8]. The transmittance of the sample was detected by a liquid nitrogen cooled charge-coupled device in conjunction with a 64-cm monochromator. The spectral resolution was $0.3 \mathrm{meV}$. Throughout the experiment, the sample was kept immersed in superfluid helium at $2 \mathrm{~K}$.

In Fig. 1(a) we show the persistent hole-burning spectra of $\mathrm{CuCl}$ nanocrystals in a glass matrix. The higher energy spectral hole (marked $M$ ) coincides with the energy of the pump beam while low-energy nonresonant holes are also seen. The low-energy holes reveal the vibrational structure in the absorption spectrum of single-sized nanocrystals that is otherwise hidden under the inhomogeneously broadened absorption profile. It may be noted that the persistent hole structure shows up as a mirror image [9] of the absorption spectrum of single-sized nanocrystals. The nonresonant spectral holes arise due to either the excitation of those nanocrystals, in which the electronic level is below the laser frequency but is excited with the assistance of phonons, or the excitation of the excited electronic states of the exciton [10]. In the former case, the energy difference between the resonant and the nonresonant holes gives the corresponding vibrational frequency in the excited state.

The low-energy side hole, referred to as the "pseudophonon wing" in the literature on PSHB of molecular systems [11], corresponds to the acoustic phonon-assisted absorption of the nanocrystal [12]. The Stokes shift of the pseudophonon wing matches the theoretical estimate of the confined acoustic phonon energy [13]. On the other 


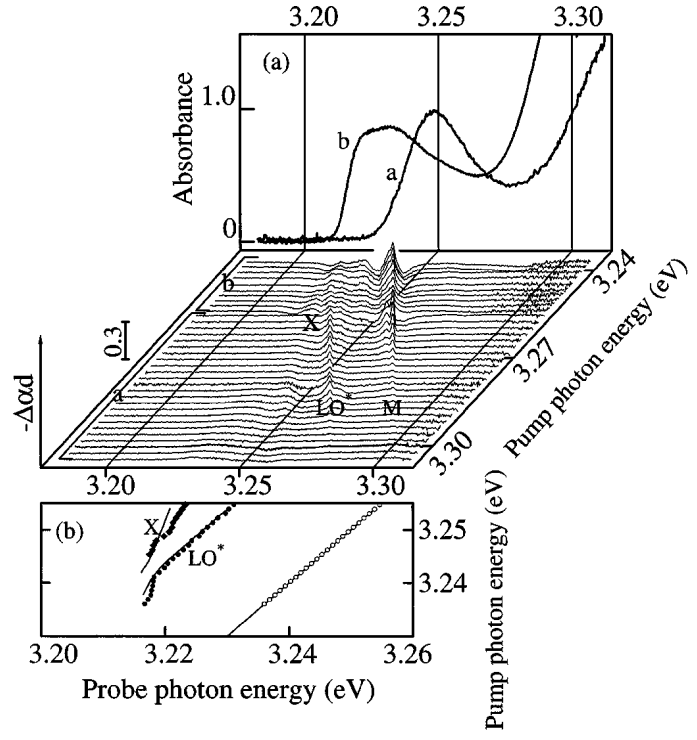

FIG. 1. (a) The inhomogeneous absorption spectrum and the persistent hole burning spectra of $\mathrm{CuCl}$ nanocrystals in glass. Data for two samples with average radii $2.5 \mathrm{~nm}$ (marked $a$ ) and $4 \mathrm{~nm}$ (marked $b$ ) are shown. The lowest energy peak for each size corresponds to the exciton ground state. Holes corresponding to the excitation of the acoustic-, TO-, and LOphonon sidebands, and the $2 S$ exciton are also seen. (b) The observed energies of the holes corresponding to the LO phonon (dots) and the $2 S$ exciton (squares), along with the theoretically calculated (renormalized) energies of LO phonon-exciton mixed modes (solid lines). The main holes (open circles) at the pump photon energies are also shown.

hand, the energy difference of $23.5 \mathrm{meV}$ between the resonant hole (M) and the next sharp nonresonant hole (marked LO* $^{*}$ ) shows an unambiguous deviation of about $2 \mathrm{meV}$ from the energy of the LO phonon $(25.6 \mathrm{meV})$ in the bulk [14]. We note that this Stokes shift is conserved as the pump frequency scans the inhomogeneous absorption profile. This is clearly seen from the straight vertical alignment of the LO* peaks in Fig. 1(a). Persistent holes corresponding to the same vibrational frequencies are dominant in the PSHB spectra of $\mathrm{CuCl}$ nanocrystals embedded in a crystalline $\mathrm{NaCl}$ matrix also. The Stokes shift of $23.5 \mathrm{meV}$ remains constant across the inhomogeneous absorption line independent of the matrix material. Thus the Stokes shift of $23.5 \mathrm{meV}$ may be definitely understood to be an intrinsic property of $\mathrm{CuCl}$ nanocrystals and we attribute it to the $\mathrm{LO}$ phonon. The softening by about $2 \mathrm{meV}$ of the LO phonon energy arises from the phonon renormalization in the excited state due to the exciton-phonon coupling, as we find from the theoretical calculation to be described later. An additional nonresonant hole (marked $X$ in Fig. 1) is seen at a lower energy which is identified to arise from the excitation of the $2 S$ state of the exciton, by comparing with our theory. These two peaks approach each other as the pump energy is reduced (i.e., as larger size nanocrystals are excited), and an incipient anticrossing of the two nonresonant holes is observed. This behavior agrees well with our theoretical calculation, as shown in Fig. 1(b).
Because persistent holes are formed as a result of the relaxation of the electronic excitation of the nanocrystals, phonon-assisted absorption which involves the emission of excited state phonons is observable through PSHB. Raman scattering and phonon-assisted luminescence, on the other hand, involve the ground state phonons. As illustrated in Fig. 2, the luminescence from the exciton ground state following the excitation of an excited state and subsequent intraband relaxation should also be observable. Thus, Stokes shifts corresponding to both the excited state and ground state phonons should appear in the luminescence spectrum.

In Fig. 3, we show the luminescence spectra for a $\mathrm{CuCl} /$ glass sample for different excitation frequencies. In addition to the Raman-like phonon-assisted luminescence with a Stokes shift of $26 \mathrm{meV}$ (marked LO), we also find a luminescence peak at $23.5 \mathrm{meV}$ (marked LO*) below the excitation energy, in agreement with the PSHB results. It is important to note that although both these luminescence peaks involve decay of the lowest exciton state, the former (LO) leaves behind a phonon in the ground state while the latter (LO*) is a zero-phonon line (see Fig. 2). The Stokes shift of the latter arises from the fact that the corresponding excitation involves creation of a phonon in the excited state. Consequently, as expected for zero-phonon lines, the LO* peak is observed only within the absorption spectrum of the sample, whereas the LO peak is seen below the absorption edge also.

In addition to the acoustic phonon sideband and the nonresonant holes mentioned earlier, we also observe a peak inside of the inhomogeneous absorption spectrum with a Stokes shift of about $20 \mathrm{meV}$, both in the persistent hole burning and luminescence spectra. The TO phonon energy in bulk $\mathrm{CuCl}$ being $20 \mathrm{meV}$, we attribute this to the TO phonon sideband.

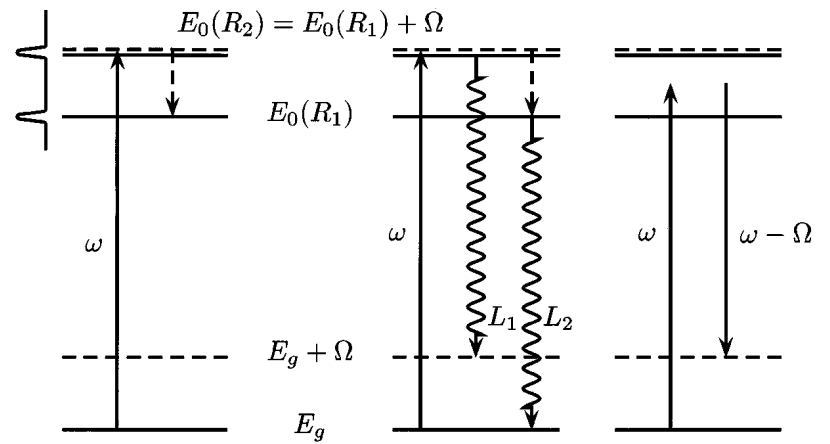

$\begin{array}{lll}\text { (a) hole burning } & \text { (b) luminescence } & \text { (c) Raman scattering }\end{array}$

FIG. 2. A schematic showing the excitation and relaxation processes involved in (a) hole burning (b) luminescence, and (c) Raman scattering. $E_{0}$ denotes the energy of the lowest exciton state, $\Omega(\tilde{\Omega})$ denotes the phonon frequency in the ground (excited) state, and $R_{1}$ and $R_{2}$ refer to radii of nanocrystals such that $E_{0}\left(R_{1}\right)+\tilde{\Omega}=E_{0}\left(R_{2}\right)$ so that both of these could be excited simultaneously. $L_{1}$ and $L_{2}$ are luminescence processes showing Stokes shifts of $\Omega$ and $\tilde{\Omega}$, respectively. 


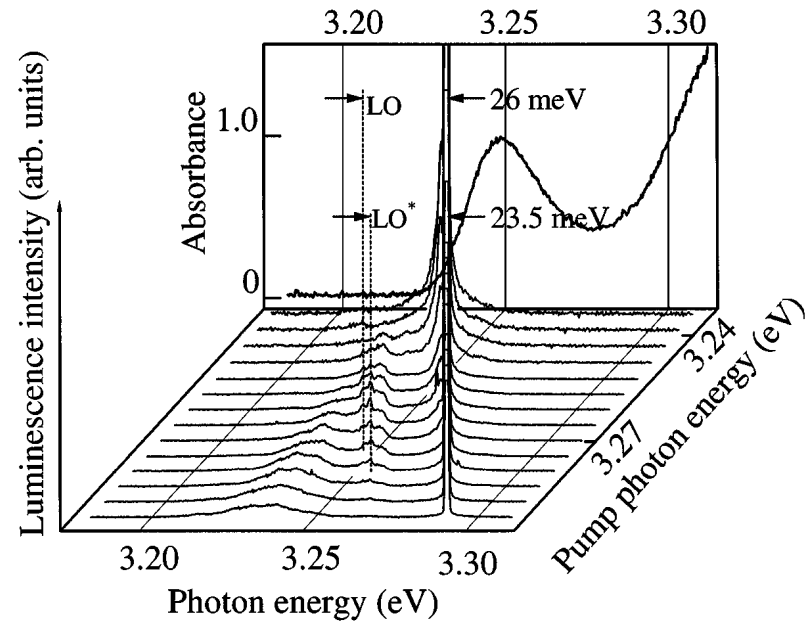

FIG. 3. The luminescence spectra of $\mathrm{CuCl}$ nanocrystals in a glass matrix (average radius $2.5 \mathrm{~nm}$ ) as the excitation frequency scans the absorption profile.

To understand the LO phonon renormalization, we consider the exciton-phonon Fröhlich Hamiltonian within a macroscopic approach for the confined LO phonons [4],

$$
\begin{aligned}
H= & \sum_{i} E_{i} c_{i}^{\dagger} c_{i}+\sum_{\nu l m} \Omega a_{\nu l m}^{\dagger} a_{\nu l m} \\
& +\sum_{i j \nu l m} \gamma_{\nu l m}^{i j} c_{i}^{\dagger} c_{j}\left(a_{\nu l m}^{\dagger}+a_{\nu l m}\right),
\end{aligned}
$$

where $c_{i}$ and $E_{i}$, respectively, denote the annihilation operator and energy for the $i$ th exciton state, $a_{\nu l m}$ is the phonon annihilation operator for the $\nu$ th phonon mode of angular momentum $l$, and $\Omega$ is the LO phonon frequency neglecting dispersion. The coupling constants $\gamma_{\nu l m}^{i j}$ are given by

$$
\begin{aligned}
\gamma_{\nu l m}^{i j}= & -e \sqrt{\frac{4 \pi \Omega}{\kappa R \xi_{\nu l}^{2}}} \int \psi_{i}^{*}\left(\mathbf{r}_{e}, \mathbf{r}_{h}\right) \\
& \times\left[u_{\nu l m}\left(\mathbf{r}_{e}\right)-u_{\nu l m}\left(\mathbf{r}_{h}\right)\right] \psi_{j}\left(\mathbf{r}_{e}, \mathbf{r}_{h}\right) d \mathbf{r}_{e} d \mathbf{r}_{h},
\end{aligned}
$$

where $\psi$ 's denote the exciton wave functions, $u_{\nu l m}=$ $j_{l}\left(\xi_{\nu l} r / R\right) Y_{l m}(\theta, \phi) / j_{l+1}\left(\xi_{\nu l}\right), \xi_{\nu l}$ 's being the zeros of the spherical Bessel function $j_{l}(r)$, and $\kappa=\epsilon_{0} \epsilon_{\infty} /\left(\epsilon_{0}-\right.$ $\left.\epsilon_{\infty}\right)$. Further, $\nabla u_{\nu l m}$ 's describe the phonon modes, and other symbols have their usual meaning.

From Eq. (2) it follows that, due to Fröhlich interaction, only the $l=0$ phonons will appear as sidebands to the $S$ excitons in the optical absorption and emission spectra. As we are interested in the phonon sidebands of the ground state exciton, here we consider only the $l=0$ phonons and excitons, and drop the subscript $\mathrm{lm}$. We calculate the exciton states in the weak confinement regime using a correlated basis set approach [15] and numerically obtain the coupling constants $\gamma_{\nu}^{i j}$.

The exciton-phonon coupling modifies the exciton and phonon energies. Going beyond the Franck-Condon ap- proximation, the change in the phonon frequency may be calculated in second order perturbation theory as

$$
\delta \Omega=-\sum_{i \neq 0} \frac{\left|\gamma_{\nu}^{0 i}\right|^{2} 2\left(E_{i}-E_{0}\right)}{\left(E_{i}-E_{0}\right)^{2}-\Omega^{2}}
$$

where 0 denotes the exciton ground state. For $R<$ $5 \mathrm{~nm}$, the phonon frequency $\Omega$ is smaller than the energy difference between the exciton ground state $(1 S)$ and the excited $S$ states. Consequently, the phonon frequency shift is negative. As the phonon energy becomes close to any of the exciton level spacings, the above treatment, however, breaks down. We rectify this limitation by calculating the phonon self-energy from the Green's function.

We consider the phonon propagator $D_{\nu \nu^{\prime}}(\omega)$ which satisfies the Dyson equation

$$
D_{\nu \nu^{\prime}}(\omega)=D_{\nu}^{(0)}(\omega)+D_{\nu}^{(0)}(\omega) \Pi_{\nu \nu^{\prime \prime}}(\omega) D_{\nu^{\prime \prime} \nu^{\prime}}(\omega),
$$

where $D_{\nu}^{(0)}(\omega)=2 \Omega /\left[\omega^{2}-(\Omega-i \delta)^{2}\right]$ is the free phonon propagator and $\Pi_{\nu \nu^{\prime}}$ is the phonon self-energy matrix. Neglecting the vertex corrections (Migdal approximation [16]), $\Pi$ is given by the polarization loop. Replacing the exciton Green's function by its free-particle value, $G_{i}^{(0)}(\omega)=\mp\left(\omega-E_{i} \mp i \delta\right)^{-1}$ [the minus (plus) sign applies for the occupied (unoccupied) exciton states] we obtain,

$$
-i \Pi_{\nu \nu^{\prime}}(\omega)=\sum_{i j} \frac{\gamma_{\nu}^{i j} \gamma_{\nu^{\prime}}^{j i}}{2 \pi} \int d \epsilon G_{i}^{(0)}(\epsilon) G_{j}^{(0)}(\epsilon+\omega) .
$$

Noting that $D_{\nu}^{(0)}$ does not depend on $\nu$, it follows that the unitary transformation that diagonalizes $\Pi$ also makes $D$ diagonal, so that Eq. (4) becomes

$$
\tilde{D}_{\mu}(\omega)^{-1}=D_{\mu}^{(0)-1}(\omega)-\tilde{\Pi}_{\mu}(\omega),
$$

where $\mu$ denotes the (renormalized) phonon normal modes that diagonalize $\Pi_{\nu \nu^{\prime}}$, and $\tilde{D}_{\mu}$ 's $\left(\tilde{\Pi}_{\mu}\right)$ denote the eigenvalues of $D(\Pi)$. The renormalized phonon frequencies, $\tilde{\Omega}_{\mu}$ are given by the poles of $\tilde{D}_{\mu}$, and satisfy

$$
\tilde{\Omega}_{\mu}^{2}-\Omega^{2}-2 \Omega \tilde{\Pi}_{\mu}(\tilde{\Omega})=0 .
$$

We calculate $\Pi_{\nu \nu^{\prime}}$ by considering ten phonon modes and ten lowest energy exciton states.

We find that a single phonon mode is strongly modified, and it is the same mode that has any significant coupling to the exciton ground state so as to be observed in the phonon-assisted absorption spectrum. In Fig. 4 we show the size dependence of the frequency of this mode, using parameters appropriate for $\mathrm{CuCl}$ nanocrystals [17]. The theory shows a phonon frequency softening of about $10 \%$ in the size range of $R=2-4 \mathrm{~nm}$. As the crystallite size is increased, the exciton-phonon coupling is found to become weaker, but the one phonon sideband of the exciton ground state approaches the $2 S$ exciton state enhancing the 


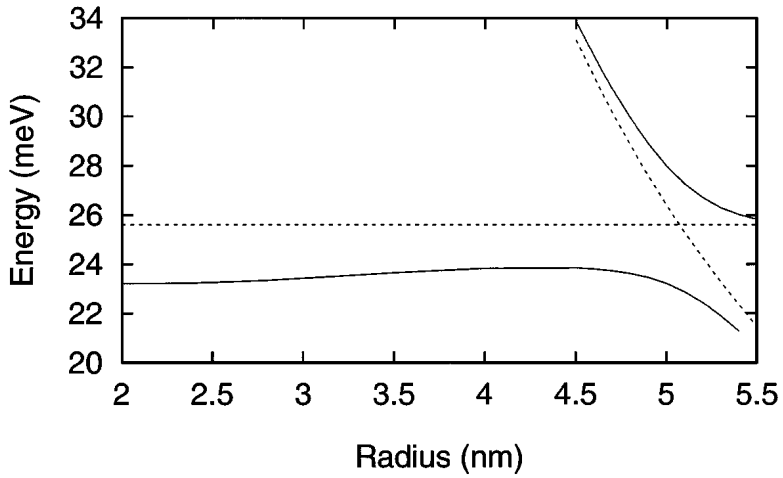

FIG. 4. The size dependence of the renormalized LO phonon frequency given by Eq. (7) for the most strongly coupled mode. The energies of the free phonon and the $2 S$ exciton state, with which the phonon shows an anticrossing at about $R=5 \mathrm{~nm}$, are also shown.

probability of phonon-assisted inelastic scattering of the exciton. It is a fortuitous cancellation of these two competing effects that leads to the relative size independence of the phonon frequency shift in a wide size range. As the crystallite size is further increased, we find an anticrossing resonance at around $R=5 \mathrm{~nm}$, i.e., when the $2 S$-exciton state becomes resonant with the phonon sideband of the exciton ground state. We observed the onset of this anticrossing in a sample containing crystallites of average radius around $4 \mathrm{~nm}$ (see Fig. 1). As noted earlier and as shown in Fig. 1(b), the calculated phonon frequency shift is in excellent agreement with the experiment.

We have assumed the LO phonon frequency in the unexcited nanocrystal to be the same as in the bulk material. In $\mathrm{CuCl}$, the phonon frequency dispersion being positive, the confinement would be expected to lead to a slight increase in the LO phonon frequency. In the size range considered by us, we estimate this increase to be about $0.5 \mathrm{meV}$. It may be noted that, in contrast to the confinement induced shift of the phonon frequency, the renormalization discussed in this paper refers to the difference between the phonon spectrum in the ground state and the electronic excited state of the nanocrystal.

We note that in many molecular systems and localized states, it is known that the vibrational frequencies in the excited state could differ appreciably from those in the ground state [7]. However, the observation of a similar effect in nanocrystals is surprising because a typical crystallite is a giant molecule that contains several thousand atoms. The reason for the observed behavior may be partly attributed to the strong ionic nature of the underlying material allowing efficient Fröhlich coupling, and also to the breakdown of the $k$-selection rules due to finite size effects. Recently, two-photon excitation experiments on $\mathrm{CuCl}$ nanocrystals have also shown a phonon frequency shift of about $2 \mathrm{meV}$ in the excited state, in good agreement with the present results [18].
In conclusion, we have revealed the sharp spectral features otherwise obscured by the inhomogeneous broadening of the absorption profile of $\mathrm{CuCl}$ nanocrystals in glass and $\mathrm{NaCl}$ matrices, by means of persistent hole burning and luminescence spectroscopies. Over a wide size range of 2 to $4 \mathrm{~nm}$, the LO phonon frequency was found to be reduced by about $2 \mathrm{meV}$ upon electronic excitation. At larger sizes, resonant mixing of the exciton and the phonon modes leads to an anticrossing behavior. A theory of the LO phonon renormalization in the presence of an exciton was developed, and the calculated phonon frequency renormalization shows excellent agreement with the experiment.

The authors thank K. Nishibayashi for help in the experiments, and L. Ramaniah for a critical reading of the manuscript.

*Permanent address: Stepanov Institute of Physics, F. Skaryna Avenue, 70 Minsk 220072, Belarus.

[1] Y.Z. Hu, S. W. Koch, M. Lindberg, N. Peyghambarian, E. L. Pollock, and F.F. Abraham, Phys. Rev. Lett. 64, 1805 (1990).

[2] M. Ikezawa, Y. Masumoto, T. Takagahara, and S. V. Nair, Phys. Rev. Lett. 79, 3522 (1997).

[3] M. Fujii, S. Hayashi, and K. Yamamoto, Appl. Phys. Lett. 57, 2692 (1990).

[4] M. C. Klein, F. Hache, D. Ricard, and C. Flytzanis, Phys. Rev. B 42, 11123 (1990).

[5] A. Tanaka, S. Onari, and T. Arai, Phys. Rev. B 47, 1237 (1993).

[6] T. Itoh, M. Nishijima, A. I. Ekimov, C. Gourdon, Al. L. Efros, and M. Rosen, Phys. Rev. Lett. 74, 1645 (1995).

[7] S. Nakajima, Y. Toyozawa, and R. Abe, The Physics of Elementary Excitations (Springer, Berlin, 1980).

[8] Y. Masumoto, S. Okamoto, T. Yamamoto, and T. Kawazoe, Phys. Status Solidi (b) 188, 209 (1995).

[9] B. M. Kharlamov, L. A. Bykovskaia, and R. I. Personov, Chem. Phys. Lett. 50, 407 (1977).

[10] Y. Masumoto, K. Sonobe, and N. Sakakura, J. Lumin. 7274, 294 (1997).

[11] J. Friedrich, J. D. Swalen, and D. Haarer, J. Chem. Phys. 73, 705 (1980).

[12] S. Okamoto and Y. Masumoto, J. Lumin. 64, 253 (1993).

[13] H. Lamb, Proc. Math. Soc. London 13, 187 (1882); T. Takagahara, J. Lumin. 70, 129 (1996).

[14] Physics of II-VI and I-VII Compounds, Semimagnetic Semiconductors, edited by O. Madelung, M. Schulz, and H. Weiss, Landolt-Börnstein, New Series, Group III, Vol. 17, Pt. b (Springer, Berlin, 1982).

[15] Y. Kayanuma, Phys. Rev. B 38, 9797 (1988).

[16] See, e.g., G. Rickayzen, Green's Functions in Condensed Matter (Academic, London, 1980).

[17] We use $m_{e}=0.5, m_{h}=1.8, \Omega=25.6 \mathrm{meV}$, and $\epsilon_{0}=$ 7.9, taken from Ref. [14], and estimate $\epsilon_{\infty}$ from the Lyddane-Sachs-Teller relation taking the TO phonon energy to be $20 \mathrm{meV}$.

[18] A. V. Baranov, S. Yamauchi, and Y. Masumoto (private communication). 